\title{
THE APPROXIMATION PROPERTY OF SOME VECTOR VALUED SOBOLEV-SLOBODECKIJ SPACES
}

\author{
CARLOS BOSCH, SALVADOR PÉREZ-ESTEVA \\ Instituto de Matemáticas, U.N.A.M. \\ Area de la Investigación Científica \\ Ciudad Universitaria \\ Mexico 04510 D.F. Mexico \\ JOAQUÍN MOTOS \\ Departamento de Matemática Aplicada \\ Universidad Politécnica de Valencia \\ Camino de Vera s/n \\ 46022 Valencia España \\ (Received February 2, 1990 and in revised form March 2, 1991)
}

\begin{abstract}
In this paper we consider the Sobolev-Slobodeckij spaces $W^{m, p}\left(\mathfrak{R}^{n}, E\right)$ where $E$ is a strict $(L F)$-space, $m \in(0, \infty) \backslash \mathrm{N}$ and $p \in[1, \infty)$. We prove that $W^{m, p}\left(\mathfrak{R}^{n}, E\right)$ has the approximation property provided $E$ has it, furthermore if $E$ is a Banach space with the strict approximation property then $W^{m, p}\left(\Re^{n}, E\right)$ has this property.
\end{abstract}

KEY WORDS AND PHRASES: Sobolev-Slobodeckij, strict $(L F)$ space, the approximation property. 1991 AMS SUBJECT CLASSIFICATION CODES. Primary 46E35, Secondary 46A14, 46A12, 46A35.

\section{INTRODUCTION.}

Let $E$ be a strict $(L F)$-space and $p \in[1, \infty) . L^{p}(E)=L^{p}\left(\mathfrak{R}^{n}, E\right)$ will denote the linear space of all Bochner measurable functions (cosets), from $\mathfrak{\Re}^{n}$ into $E$ such that

$$
\|f\|_{p}=\left(\int_{x}\|f(x)\|^{p} d x\right)^{1 / p}<\infty,
$$

for every $\|\cdot\| \in \operatorname{cs}(E)$, where $\operatorname{cs}(E)$ denotes the set of all continuous seminorms on $E$. We provide $L^{p}(E)$ with the topology generated by the family of seminorms $\left\{\|\|_{p}:\|\cdot\| \in \operatorname{cs}(E)\right\}$, which makes it a sequentially complete locally convex space $\left[3\right.$, p. 122]. We shall consider $L^{P}(E)$ canonically embedded in the space $\mathcal{D}^{\prime}(E)=\mathcal{D}^{\prime}\left(\Re^{n}, E\right)$ of vector valued distributions. For $q \in \mathbb{N}, W^{q, p}(E)=W^{q, p}\left(\Re^{n}, E\right)$ will denote the Sobolev space consisting of all functions $f \in L^{p}(E)$ such that $D^{\alpha} f \in L^{p}(E)$ for every $\alpha \in \mathbb{N}^{n}$ with $|\alpha| \leq q$ and provided with the family of seminorms:

$$
\|f\|_{q, p}-\left(\sum_{|\alpha| \leqslant q}\left(\left\|D^{\alpha} f\right\|_{p}\right)^{p}\right)^{1 / p}
$$

$D^{\alpha} f$ denotes the $\alpha$-partial derivative of $f$ in the sense of distributions and \|\|$\in \operatorname{cs}(E)$. If $g$ is a function defined in $\mathfrak{A}^{n}$, with values in $\mathrm{C}$ or in $E$, we define 


$$
H^{*} g(x, y)=\frac{g(x)-g(y)}{|x-y|^{1 / p+\lambda}} \text { for } \lambda \in[0,1] \text {. }
$$

From now on $m \in(0, \infty) \backslash \mathbf{N}, \lambda=m-[m]$. The Sobolev-Slobodeckij space $W^{m, p}(E)-W^{m, p}\left(\Re^{n}, E\right)$ is defined by

$$
W^{m, p}(E)=\left\{f \in W^{[m] p}(E): H^{*} D^{\alpha} f \in L^{p}\left(\Re^{n} \times \Re^{n}, E\right),|\alpha|-[m]\right\} .
$$

We provide $W^{m, p}(E)$ with the topology generated by the family of seminorms:

$$
\|f\|_{m, p}=\left(\|f\|_{[m b p}^{p}+\sum_{\mid \alpha-[m]}\left\|H^{*} D^{\alpha} f\right\|_{p}^{p}\right)^{1 / p},
$$

for every $\|\cdot\| \in \operatorname{cs}(E)$. Then the space $W^{m, p}(E)$ is a sequentially complete locally convex space [6].

Let $L_{c}(E)$ be the space of linear continuous operators in $E$ with the topology of uniform convergence on precompact subsets of $E$. We say that $E$ has the (strict) approximation property, see [5, p. 232] or [10], if the continuous linear operators of finite rank are (strictly) dense in $L_{c}(E)$. (A subset $A$ of a locally convex space $F$ is quasi-closed if it contains all the accumulation points in $F$ of its bounded subsets. The quasi-closure of $A$, denoted $\hat{A}$ is the intersection of all quasi-closed sets of $F$ that contain $A$. We of course have $\hat{A} \subset \bar{A}$ but in general $\hat{A}=\bar{A}$. If $\hat{A} \supset B$ then $A$ is strictly dense in $B$ [9, pp. 91-92]. See [10] for further information of the strict approximation property.)

Now it is well known that the Sobolev spaces $W^{m, p}\left(\mathfrak{A}^{n}\right), 1<p<\infty, m \in \mathbb{N}$ are isomorphic to the Lebesgue spaces $L^{P}(0,1)$. Pelczynski and Senator [8] proved that this is also true in the anisotropic case. The Sobolev-Slobodeckij spaces $W^{m, p}\left(\mathfrak{P}^{n}\right), 1<p<\infty, m \in(0, \infty) \backslash \mathbb{W}$ are isomorphic to the sequence spaces $l^{p}\left[11\right.$, pp. 273-290]. For $m \in[0, \infty)$ and $1<p<\infty$ the Banach spaces $W^{m, p}\left(\mathfrak{A}^{m}\right)$ have an unconditional Schauder base and so the approximation property. The Sobolev spaces (isotropic and anisotropic) with values in a Fréchet space $E$ and domain on $\mathfrak{R}^{n}$ have the approximation property provided $E$ has it and $1 \leq p<\infty[7]$.

In this paper we prove that the Sobolev-Slobodeckij spaces $W^{m, p}(E), 1 \leq p<\infty, m \in(0, \infty) \backslash \mathbb{N}, E$ a strict $(L F)$-space have the approximation property provided $E$ has it, furthermore $W^{m, P}(E)$ has the strict approximation property if $E$ is Banach space and has the strict approximation property. The proof is based on Schwartz argument to prove that some scalar spaces of distributions have the strict approximation property [10, pp. 9-10]. In Section 1 we prove that $W^{m, p}(E)$ has the regularizing approximation property $\left[10\right.$, p. 8]. In Section 2 we shall prove that $W^{m, p}(E)$ has the truncating approximation property [10, p. 7]. Finally in the last section we prove that $\mathcal{D}(E)$ is strictly dense in $W^{m, p}(E)$ and that $W^{m, p}(E)$ has the (strict) approximation property provided $E$ (Banach space) has the (strict) approximation property.

\section{2. $W^{m, P}(E)$ has the Regularizing Approximation Property}

Let $\mathscr{D}^{\left(\mathfrak{R}^{n}\right)}$ be the standard space of test functions in $\mathfrak{R}^{n}$. We say that a sequence $\left\{\eta_{j}\right\}_{j \in \mathbb{n}} \subset\left(\mathfrak{R}^{n}\right)$ is regularizing if
1) $\eta_{j}(x) \geq 0$
for all $x \in \mathfrak{R}^{*}$ and $j \in \mathbb{H}$,
2) $\eta_{j}(x)=0$
if $|x|>\varepsilon_{j}$ and $\varepsilon_{j} \rightarrow 0^{+}$,
3) $\int_{x^{\infty}} \eta_{j}(x) d x=1$
for all $j \in \mathbf{H}$.

For $\eta \in \mathcal{D}^{\left(\Re^{n}\right)}$ and $f \in L^{P}(E)$ we define the convolution 


$$
\eta^{*} f(x)=\int_{\mathscr{P}^{n}} \eta(z) f(x-z) d z
$$

Since the space of continuous functions with compact support of $\mathfrak{R}^{n}$ into $E$ is dense in $L^{p}(E)$ and using arguments similar to the scalar case we have:

a) $\eta^{*} f \in \varepsilon(E)$ (space of all $E$-valued infinitely differentiable functions on $\left.\Re^{n}\right), D^{\alpha}\left(\eta^{*} f\right)=\left(D^{\alpha} \eta\right){ }^{*} f$ if $\alpha \in \boldsymbol{B}^{n}$ and for any \|\|$\in \operatorname{cs}(E),\left\|\eta^{*} f\right\|_{p} \leq\|\eta\|_{L^{1}\left(R^{n}\right)}\|f\|_{p}$,

b) $\eta^{*} f \in \mathcal{D}_{L^{p}}(E)-\left\{f \in \varepsilon(E): D^{\alpha} f \in L^{p}(E)\right.$ for all $\left.\alpha \in \mathbb{N}^{*}\right\}$,

c) $\left(\eta_{j}^{*} f\right)_{j \in \pi}$ converges to $f$ in $L^{p}(E)$.

In this section we prove that

$$
f_{j}=\eta_{j}^{*} f \rightarrow f \text { in } W^{m, p}(E),
$$

uniformly in precompact subsets of $W^{m, p}(E)$.

LEMMA 2.1 Let \|\|$\in c s(E)$. For $f \in L^{p}(E)$ and $F \in L^{p}\left(\Re^{n} \times \Re^{n}, E\right)$, define

$$
\Phi_{f}(z)-\left(\int_{T^{*}}\left\|\tau_{z} f-f\right\|^{p} d x\right)^{1 / p}-\left\|\tau_{z} f-f\right\|_{p}
$$

and

$$
\Psi_{F}(z)=\left(\int_{\mathscr{T}^{n}} \int_{\mathscr{T}^{n}}\left\|\tau_{(z, z)} F-F\right\|^{p} d x d y\right)^{1 / p}=\left\|\tau_{(z, z)} F-F\right\|_{p},
$$

where $\tau_{z} f(x)=f(x-z)$ and $\tau_{(z, z)} F(x, y)=F(x-z, y-z)$. If $\mathcal{A} \subset L^{p}(E)$ (resp. $\mathcal{B} \subset L^{p}\left(\Re^{n} \times \Re^{n}, E\right)$ ) is precompact then $\left\{\Phi_{f}\right\}_{f \in \mathfrak{A}}$ (resp. $\left.\left\{\Psi_{F}\right\}_{F \in g}\right)$ is precompact in the space $C^{b}\left(\mathfrak{P}^{n}\right)$ of bounded continuous complex functions provided with the supremum norm.

PROOF. We prove it for $\left\{\Phi_{f}\right\}_{f \in \Omega}$, the other part is similar. First observe that the operator defined by $g \rightarrow \tau_{z} g$ is continuous in $L^{p}(E)$, the proof is the same as in the case $E=\mathrm{C}$. We also have:

$$
\left|\Phi_{f}(z)-\Phi_{g}(z)\right|=\left|\left\|\tau_{z} f-f\right\|_{p}-\left\|\tau_{z} g-g\right\|_{p}\right| \leq\left\|\tau_{z}(f-g)\right\|_{p}+\|f-g\|_{p}=2\|f-g\|_{p} .
$$

Hence the mapping $\Phi: L^{p}(E) \rightarrow C^{b}\left(\Re^{n}\right), f \rightarrow \Phi_{f}$, is uniformly continuous and the lemma follows.

LEMMA 2.2. If $f \in L^{P}(E)$ then the function $F: \mathfrak{A}^{n} \times \mathfrak{N}^{n} \rightarrow E$ defined by $F(x, y)-f(x-y)$ is Bochner-measurable.

PROOF. Since $E$ is a strict $(L F)$-space, $E=$ ind $\lim E_{k}$ and $f \in L^{p}(E)$ then using the same argument as in $\left[2\right.$, p. 255] there exists a $k \in H$ such that $f(x) \in E_{k}$ almost everywhere. So we can assume that $f$ is a Bochner-measurable function from $\mathfrak{P}^{n}$ to $E_{k}$ and using Pettis measurability theorem, valid for Fréchet spaces, we have that $F$ is Bochner measurable from $\mathfrak{R}^{n} \times \mathfrak{A}^{n}$ in $E_{k}$ so $F$ is Bochner-measurable from $\mathfrak{R}^{n} \times \mathfrak{R}^{n}$ into $E$.

LEMMA 2.3. Let $\|\cdot\| \in c s(E), \eta \in \mathcal{D}\left(R^{n}\right)$ and $f \in L^{p}(E)$ such that $H^{*} f \in L^{p}\left(\Re^{n} \times \Re^{n}, E\right)$ then $H^{*}\left(\eta^{*} f\right) \in L^{P}\left(\Re^{*} \times \Re^{*}, E\right)$ and

$$
\left\|H^{*}\left(\eta^{*} f\right)\right\|_{P} \leq\|\eta\|_{L^{1}\left(\pi^{*}\right)}\left\|H^{*} f\right\|_{P}
$$


PROOF. As in Lemma 2.2 we can establish the measurability of the functions $(x, y, z) \rightarrow f(x-z)$, $(x, y, z) \rightarrow f(y-z)$ and $(x, y, z) \rightarrow \frac{|\eta(z)||f(x-z)-f(y-z)|^{p}}{|x-y|^{p+p^{\lambda}}}$. Now,

$$
\begin{aligned}
& \left\|H^{*}\left(\eta^{*} f\right)\right\|_{P}=\left\{\int_{\mathscr{T}^{*}} \int_{\mathscr{T}^{*}}\left|\int_{\mathscr{T}^{*}} \frac{\eta(z)(f(x-z)-f(y-z))}{|x-y|^{n / p+\lambda}} d z\right|^{p} d x d y\right\}^{1 / p} \\
& \leq\left\{\int_{\mathscr{T}^{*}} \int_{\boldsymbol{N}^{*}}\left(\int_{\boldsymbol{T}^{*}} \frac{|\eta(z)| \| f(x-z)-f(y-z)) \|}{|x-y|^{n / p+\lambda}} d z\right)^{p} d x d y\right\}^{1 / p} \\
& \leq \int_{\mathscr{T}^{*}}|\eta(z)|\left\{\int_{\mathscr{N}^{*}} \int_{\mathscr{N}^{*}} \frac{\|f(x-z)-f(y-z)\|^{p}}{|(x-z)-(y-z)|^{n+p^{\lambda}}} d x d y\right\}^{1 / p} d z
\end{aligned}
$$

Minkowski's inequality

$$
=\|\eta\|_{L^{\prime}\left(x^{\prime}\right)}\left\|H^{*} f\right\|_{P}
$$

LEMMA 2.4. Let $f \in L^{p}(E)$ be such that $H^{*} f \in L^{P}\left(\mathfrak{P}^{n} \times \mathfrak{R}^{n}, E\right)$ then for every $\|\cdot\| \in c s(E)$ we have the estimates

$$
\begin{gathered}
\left\|f_{j}-f\right\|_{p} \leq \int \eta_{j}(z) \Phi_{f}(z) d z, \\
\left\|H^{*} f_{j}-H^{*} f\right\|_{P} \leq \int \eta_{j}(z) \Psi_{H^{*}}(z) d z,
\end{gathered}
$$

where $\left\{\boldsymbol{\eta}_{j}\right\}_{j \in \mathbb{x}}$ is a regularizing sequence.

PROOF. The proof is a direct application of Minkowski's inequality.

THEOREM 2.5. $W^{m, p}(E)$ has the regularizing approximation property.

PROOF. By Lemma 2.2 we have $\eta^{*} f \in W^{m, p}(E)$ provided $\eta \in \mathcal{D}\left(\mathbf{R}^{*}\right)$ and $f \in W^{m, p}(E)$. Further

$$
\left\|\eta^{*} f\right\|_{m, p} \leq\|\eta\|_{L^{1}\left(m^{*}\right)}\|f\|_{m, p} .
$$

From (2.1) and (2.2) we have

$$
\begin{gathered}
\left\|D^{\alpha} f_{j}-D^{\alpha} f\right\|_{p} \leq \int_{\mathcal{Y}^{*}} \eta_{j}(z) \Psi_{D^{\alpha} f}(z) d z, \\
\left\|h^{*} D^{\beta} f_{j}-H^{*} D^{\beta} f\right\|_{P} \leq \int_{\boldsymbol{X}^{*}} \eta_{j}(z) \Psi_{H^{*} D^{\beta} f}(z) d z,
\end{gathered}
$$

for $|\alpha| \leq[m]$ and $|\beta|-[m]$.

Let $\mathcal{B} \subset W^{m, p}(E)$ be precompact, then $\left\{D^{\alpha} f: f \in \mathcal{B}\right\}$ is precompact in $L^{P}(E)$, and $\left\{\Phi_{D^{\alpha} f}: f \in \mathcal{B}\right\}$ is precompact in $C^{b}\left(\mathfrak{R}^{n}\right)$, hence equicontinuous in compact subsets of $\mathfrak{P}^{n}$. It follows that

$$
\int_{x^{*}} \eta_{j}(z) \Phi_{D^{\alpha} f}(z) \rightarrow \Phi_{D^{\alpha} f}(0)=0,
$$

uniformly in B. Similarly

$$
\int_{r^{*}} \eta_{j}(z) \Psi_{k^{*} D^{p} f}(z) d z \rightarrow 0,
$$

uniformly in $B$. The theorem follows from (2.3) and (2.4). 


\section{3. $W^{m, p}(E)$ has the Truncating Approximation Property}

Let $\mathcal{B}\left(\mathfrak{H}^{n}\right)$ be the space of all bounded $C^{\infty}$ complex functions $f$ defined in $\mathfrak{R}^{n}$ such that

$$
\rho_{k}(f)=\sup _{\substack{|\alpha| \leq k \\ x \in \Re^{n}}}\left|D^{\alpha} f(x)\right|<\infty, \quad k \in \mathbf{N} \text {. }
$$

We provide $\mathcal{B}\left(\mathfrak{\Re}^{n}\right)$ with the topology defined by the family of seminorms $\left\{\rho_{k}\right\}_{k \in \boldsymbol{x}}$. In this section we prove that $W^{m, p}(E)$ has the truncating approximation property, that is, given a sequence $\left\{\theta_{j}\right\}_{j \in \boldsymbol{n}} \subset \mathcal{D}\left(\Re^{n}\right)$ such that $\lim _{j \rightarrow \infty} \theta_{j}=1$ in $\varepsilon\left(\mathfrak{R}^{n}\right)$ the space of all $C^{\infty}$ complex functions in $\mathfrak{R}^{n}$, and such that $\left\{\theta_{j}\right\}_{j \in \boldsymbol{N}}$ is bounded in $\mathcal{B}\left(\Re^{n}\right)$, then $\lim _{j \rightarrow \infty} \theta_{j} f=f$ uniformly in precompact subsets of $W^{m, p}(E)$.

LEMMA 3.1. Let $\theta \in \mathcal{B}\left(\Re^{n}\right), f \in W^{1, p}(E)$ and $\|\cdot\| \in c s(E)$. Then there exists $c>0$ depending on $n, p$ and $\lambda$ only, such that

$$
\left\|H^{*}(\theta f)\right\|_{p} \leq c \rho_{1}(\theta)\|f\|_{1, p} .
$$

PROOF. Let $f \in W^{1, p}(E) \cap \varepsilon(E)$ then

$$
\theta(x) f(x)-\theta(y) f(y)=\int_{0}^{1} g^{\prime}(s) d s,
$$

with

$$
g(s)=h(s x+(1-s) y) \text { and } h=\theta f
$$

By Grothendieck's lemma [9], $g \in \varepsilon(\Re, E)$. An easy calculation shows that

$$
\int_{0}^{1} g^{\prime}(s) d s=\sum_{i=1}^{n} \int_{0}^{1}\left(\frac{\partial}{\partial x_{i}} h(s x+(1-s) y) d s\left(x_{i}-y_{i}\right) .\right.
$$

Hence

$$
\|\theta(x) f(x)-\theta(y) f(y)\|^{p} \leq n^{p / p^{\prime}}|x-y|^{p} \sum_{i=1}^{n} \int_{0}^{1}\left\|\frac{\partial}{\partial x_{i}} h(s x+(1-s) y)\right\|^{p} d s,
$$

where $\frac{1}{p}+\frac{1}{p^{\prime}}=1$.

We have

$$
\int_{\mathscr{T}^{*}} \int_{\mathscr{R}^{*}}\left\|H^{*}(\theta f)\right\|^{p} d x d y=\int_{|x-y|>1}\left\|H^{*}(\theta f)\right\|^{p} d x d y+\int_{|x-y|<1}\left\|H^{*}(\theta f)\right\|^{p} d x d y-I_{1}+I_{2} .
$$

Now

$$
I_{1} \leq 2^{p / p^{\prime}} \int_{|x-y|>1} \frac{|\theta(x)|^{p}\|f(x)\|^{p}}{|x-y|^{n+p \lambda}} d x d y \leq 2^{p / p^{\prime}} \rho_{0}(\theta)^{p}\left(\int_{|z|>1} \frac{d z}{|z|^{n+p \lambda}}\right)\|f\|_{p}^{p}=c_{1} \rho_{0}(\theta)^{p}\|f\|_{p}^{p} ;
$$




$$
\begin{aligned}
I_{2} & \leq \sum_{i=1}^{n} n^{p / p^{\prime}} \int_{0}^{1} \int_{|x-y|<1} \int_{\left.\right|^{\prime}} \frac{\left\|\frac{\partial}{\partial x_{i}} h(s x+(1-s) y)\right\|^{p}}{|x-y|^{n+p(\lambda-1)}} d x d y d s \\
& =\sum_{i=1}^{n} n^{p / p^{\prime}} \int_{0}^{1} \int_{q^{n}} \int_{|z|=1} \frac{\left\|\frac{\partial}{\partial_{i}} h(x-s z)\right\|^{p}}{|z|^{n+p(\lambda-1)}} d z d x d s \\
& =\sum_{i=1}^{n} n^{p / p^{\prime}} \int_{0}^{1} \int_{|z|=1} \frac{1}{|z|^{n+p(\lambda-1)}} \int_{F^{*}}\left\|\frac{\partial}{\partial_{i}} h(x+s z)\right\|^{p} d x d z d s \\
& \leq c_{2} \rho_{1}(\theta)^{p}\|f\|_{p}^{p} .
\end{aligned}
$$

From (3.2) and (3.3) we obtain

$$
\left\|H^{*}(\theta f)\right\|_{p} \leq c \rho_{1}(\theta)\|f\|_{1, p} .
$$

Now let $f \in W^{1, p}(E)$ and $\left\{\eta_{j}\right\}_{j \in}$ be a regularizing sequence in $\mathcal{D}\left(\Re^{n}\right)$. We can assume (taking a subsequence if necessary) that $\left\|\eta_{j}{ }^{*} f(x)-f(x)\right\|$ converges to zero almost everywhere in $\mathfrak{R}^{n}$. Let $f_{j}=\eta_{j}{ }^{*} f$, then

$$
\left\|H^{*}\left(\theta f_{j}\right)\right\|_{p} \leq c \rho_{1}(\theta)\left\|f_{j}\right\|_{1, p} \leq c \rho_{1}(\theta)\|f\|_{1, p} .
$$

Taking the limit as $j$ tends to infinity we obtain, by Fatou's lemma,

$$
\left\|H^{*}(\theta f)\right\|_{p} \leq c \rho_{1}(\theta)\|f\|_{1, p} .
$$

LEMMA 3.2. Let $\|\cdot\| \in \operatorname{cs}(E)$. Then there exists $c>0$ depending on $n, p$ and $\lambda$ only such that

$$
\|\theta f\|_{m, p} \leq c \rho_{[m]+1}(\theta)\|f\|_{m, p},
$$

for every $\theta \in \mathcal{B}\left(\mathfrak{R}^{n}\right)$ and $f \in W^{m, P}(E)$.

PROOF. We have

$$
\|\theta f\|_{m, p}^{p}=\sum_{|\alpha|-[m]} \iint \frac{\left\|D^{\alpha}(\theta f)(x)-D^{\alpha}(\theta f)(y)\right\|^{p}}{|x-y|^{n+\lambda p}} d x d y+\|\theta f\|_{[m] p}^{p} .
$$

We clearly have

$$
\|\theta f\|_{[m b p} \leq c_{1} \rho_{[m]}(\theta)\|f\|_{[m b p},
$$

by Lemma 3.1

$$
\left\|H^{*} D^{\beta} \theta D^{\alpha} f\right\|_{p} \leq c_{2} \Omega_{[m]+1}(\theta)\|f\|_{[m\} p},
$$

for $|\alpha|+|\beta|-[m]$ and $|\alpha|<[m]$.

Finally if $|\alpha|-[m]$

$$
\int_{x^{*}} \int_{x^{*}}\left\|H^{*}\left(\theta D^{\alpha} f\right)\right\|^{p} d x d y=\int_{|x-y|>1} \int_{|x-y|=1}\left\|H^{*}\left(\theta D^{\alpha} f\right)\right\|^{p} d x d y+\int_{\mid} \int_{\mid{ }^{*}}\left(\theta D^{\alpha} f\right) \|^{p} d x d y .
$$

As in Lemma 3.1 we can bound the first integral by $c_{3} \rho_{0}(\theta)\|f\|_{[m\} p}$ and

$$
\begin{aligned}
& \iint_{|x-y|<1}\left\|H^{*}\left(\theta D^{\alpha} f\right)\right\| d x d y \leq 2^{p / p^{\prime}} \iint_{|x+y|<1} \frac{|\theta(x)|^{p}\left\|D^{\alpha} f(x)-D^{\alpha} f(y)\right\|^{p}}{|x-y|^{n+\lambda p}} d x d y \\
& +2^{p / p^{\prime}} \int_{|x-y|=1} \frac{\left\|D^{\alpha} f(x)\right\||\theta(x)-\theta(y)|^{p}}{|x-y|^{n+\lambda_{p}}} d x d y,
\end{aligned}
$$

and since $|\theta(x)-\theta(y)| \leq \rho_{1}(\theta)|x-y|$, we have 


$$
\iint_{|x-y| \leq 1} \| H^{*}\left(\theta D^{\alpha} f\left\|^{p} d x d y \leq C_{4} \rho_{0}(\theta)\right\| f \|_{m, p} .\right.
$$

The lemma follows form (3.5), (3.6), (3.7), (3.8), (3.9) and Leibnitz's formula.

LEMMA 3.3. Let $\|\cdot\| \in c s(E)$ and $\left\{f_{n}\right\}_{n \in \mathbb{M}} \subset L^{p}(E)$. If $\left\{\left\|f_{n}\right\|\right\}_{n \in \mathbb{X}}$ and $\left\{\left\|H^{*} f_{n}\right\|\right\}_{n \in X}$ are Cauchy sequences in $L^{p}\left(\mathfrak{R}^{n}\right)$ and $L^{P}\left(\mathfrak{R}^{n} \times \mathfrak{R}^{n}\right)$ respectively, then for any truncating sequence $\left\{\theta_{n}\right\}_{n} \in \mathbb{x}$ we have $\left\|H^{*}\left(1-\theta_{n}\right) f_{n}\right\|_{p} \rightarrow 0$.

PROOF. Let $m>0$ and $\phi_{n}=1-\theta_{n} ; n \in \mathbf{N}$

$$
\begin{aligned}
& \left\|H^{*} \phi_{n} f_{n}\right\|_{p} \leq\left[\iint_{x^{*} \times \mathscr{S}^{*}}\left\|f_{n}(x)\right\|^{p}\left|H^{*} \phi_{n}(x, y)\right|^{p} d x d y\right]^{1 / p} \\
& +\left[\iint_{N^{*} \times T^{n}}\left|\phi_{n}(x)\right|^{p}\left\|H^{*} f_{n}(x, y)\right\|^{p} d x d y\right]^{1 / p} .
\end{aligned}
$$

Let $I_{1}$ and $I_{2}$ denote the first and second brackets respectively.

Since $\left\{\left\|H^{*} f_{n}\right\|\right\}$ is a Cauchy sequence in $L^{p}\left(\mathfrak{R}^{n} \times \mathfrak{R}^{n}\right)$ and $\phi_{n} \rightarrow 0$ pointwise boundedly, we have that $I_{2} \rightarrow 0$ as $n$ goes to infinity. $I_{1}^{p}$ can now be written as the sum of three integrals

$$
\begin{aligned}
& J_{1}=\iint_{\substack{|x| \leq M \\
|x-y|<1}}\left\|f_{n}(x)\right\|^{P}\left|H^{*} \phi_{n}(x, y)\right|^{p} d x d y, \\
& J_{2}=\iint_{\substack{|x|>N \\
|x-y| 1=1}}\left\|f_{n}(x)\right\|^{P}\left|H^{*} \phi_{n}(x, y)\right|^{p} d x d y, \\
& J_{3}=\int_{|x-y|>1}\left\|f_{n}(x)\right\|^{p}\left|H^{*} \phi_{n}(x, y)\right|^{p} d x d y .
\end{aligned}
$$

Now as in the proof of Lemma 3.2 we can see that

With similar arguments we see that

$$
\begin{aligned}
& J_{1} \leq\left(\int_{|x| \leq M}\left\|f_{n}\right\|^{p} d x\right) \sup _{\substack{|\xi|<M+1 \\
|\alpha|<1}}\left|D^{\alpha} \phi_{n}(\xi)\right|^{p} \int_{|z|^{2}=1} \frac{d z}{|z|^{p+(\lambda-1) p}}, \\
& J_{2} \leq\left(\int_{|x|>M}\left\|f_{n}\right\|^{p} d x\right) \sup _{|\xi| \in \Re^{n}}\left|D^{\alpha} \phi_{n}(\xi)\right|_{\mid z}^{p} \int_{\mid z=1} \frac{d z}{|z|^{\alpha+(\lambda-1) p}} .
\end{aligned}
$$

$$
J_{3} \leq 2^{p / p^{\prime}} \int_{Y^{*}}\left\|f_{n}(x)\right\|^{p}\left|\phi_{n}(x)\right|^{p} d x \int_{|z|>1} \frac{d z}{|z|^{n+\lambda_{p}}}+2^{p / p^{\prime}} \int_{\mathscr{Y}^{*}}\left\|f_{n}(x)\right\|^{p} \int_{|x-y|>1} \frac{\left|\phi_{n}(y)\right|^{p}}{|x-y|^{n+\lambda_{p}}} d y d x .
$$

Since $\left\|f_{n}\right\|$ is a Cauchy sequence in $L^{P}\left(\Re^{n}\right)$, taking $M$ large enough, all the terms involving $\int_{|x|>M}\left\|f_{n}\right\|^{P} d x$ can be made arbitrarily small, all the other terms tend to zero by Lebesgue's dominated converge theorem and the fact that $\phi_{n}$ converges to zero uniformly in $\{x:|x| \leq M\}$.

THEOREM 3.4 $W^{m, r}(E)$ has the truncating approximation property.

PROOF. Let $\left\{\theta_{j}\right\}_{j \in \mathbb{x}}$ be a truncating sequence, $\|\cdot\| \in \operatorname{cs}(E)$ and $\Re \subset W^{m, p}(E)$ be precompact. Assume that there exists a sequence $\left\{f_{n}\right\}_{n \in n} \subset \mathcal{A}$ such that

$$
\left\|\left(1-\theta_{j n}\right) f_{n}\right\|_{m, p} \geq \varepsilon,
$$

for some $\varepsilon>0$ and some subsequence $\left\{\theta_{j_{n}}\right\}_{n \in}$. Since $\lambda$ is precompact we can assume that $\left\{\left\|D^{\alpha} f_{n}\right\|\right\}_{n \in \Perp}$ and $\left\{\left\|H^{*} D^{\alpha} f_{n}\right\|\right\}_{n \in \boxplus}$ are Cauchy sequences in $L^{P}\left(\mathfrak{P}^{n}\right)$ and $L^{P}\left(\mathfrak{P}^{n} \times \mathfrak{P}^{n}\right)$ respectively, for $|\alpha| \leq[m]$. Then 
by Lemma 3.3 we have

$$
\left\|H^{*}\left(1-\theta_{j n}\right) D^{\alpha} f_{n}\right\|_{p} \rightarrow 0 \text { for }|\alpha|=[m] .
$$

If $\beta<\alpha$ and $|\alpha|=[m]$ we also have $\left\|H^{*} D^{\alpha-\beta}\left(1-\theta_{j n}\right) D^{\beta} f_{n}\right\|_{p} \rightarrow 0$ which contradicts 3.10 .

It follows that $\left\|\left(1-\theta_{j}\right) f\right\|_{m, p} \rightarrow 0$ uniformly on $\lambda$.

\section{The Approximation Property of $W^{m p}(E)$}

Let $\left\{\eta_{i}\right\}_{i \in \sharp}$ and $\left\{\boldsymbol{\theta}_{j}\right\}_{j \in \boldsymbol{x}}$ be regularizing and truncating sequences in $\mathcal{D}\left(\mathfrak{P}^{n}\right)$. For each $i$ and $j$ define the operators in $W^{m, p}(E)$

$$
\left\{\eta_{i}\right\}(f)=\eta_{i}^{*} f
$$

and

$$
\left[\theta_{j}\right](f)=\theta_{j} f .
$$

By Theorems 2.5 and 3.4, we have that the identity $I$ in $W^{m, p}(E)$ is in the quasiclosure of $\left\{\left\{\eta_{j}\right\} \circ\left[\theta_{j}\right]\right.$ : $i, j \in \mathbb{N}\}$ in $\mathcal{L}_{c}\left(W^{m, p}(E)\right)$; note that the previous set is bounded in $\mathcal{L}_{c}\left(W^{m, p}(E)\right)$. In particular $\mathcal{D}(E)$ is dense in $W^{m, p}(E)$, where $\mathcal{D}(E)$ is the subspace of $\varepsilon(E)$ whose elements have compact support.

L. Schwartz in [10] uses a weaker definition of the approximation property, strict approximation property, than Grothendieck's definition since it is built on the bornology of all the absolutely convex compact sets. In the next theorem, we shall use the following proposition proved in [10, p. 7].

PROPOSITION 4.1 Let $E$ be a locally convex space, $F$ is a linear subspace of $E$ with a locally convex topology finer than the topology inherited from $E$. If the identity $I$ is an accumulation point (strict) of $\mathcal{L}(E, F)$ in $\mathcal{L}_{c}(E)$ and if $F$ has the (strict) approximation property then $E$ also has the (strict) approximation property.

THEOREM 4.2 1. If $E$ is a strict $(L F)$-space and has the approximation property then $W^{m, p}(E)$ has this property.

2. If $E$ is a Banach space with the strict approximation property then $W^{m, p}(E)$ has this property.

PROOF. 1. Let $\left(\eta_{j}\right)_{j \in \Pi}$ and $\left(\theta_{j}\right)_{j \in \mathbb{m}}$ be regularizing and truncating sequences in $\mathcal{D}\left(\mathfrak{P}^{n}\right)$ respectively. Consider the following diagram:

$$
\begin{aligned}
T & \rightarrow \eta_{k}^{*} \theta_{j} T \\
W^{m, p} \stackrel{k}{\rightarrow} L^{p}(E) \rightarrow \mathcal{D}^{\prime}(E) & \rightarrow \mathcal{D}(E), \\
f & \rightarrow\left\{\phi \rightarrow \int_{\mathcal{x}^{*}} \phi f\right\},
\end{aligned}
$$

where $h$ is the canonical injection, and $\mathcal{D}(E)$ has the inductive limit topology. Note that all functions of the diagram are continuous. This implies that

$$
\left\{\left\{\eta_{k}\right\} \circ\left[\theta_{j}\right]: j, k, \in \mathbb{N}\right\} \subset \mathcal{L}\left(W^{m, p}(E), \mathcal{D}(E)\right),
$$

and by Theorems 2.3 and 3.4 the identity $I$ belongs to the quasiclosure of $\mathcal{L}\left(W^{m, P}(E), \mathcal{D}(E)\right)$ in the space $\mathcal{L}_{c}\left(W^{m, p}(E)\right)$. Now $\mathcal{D}(E)$ is topologically isomorphic to a numerable locally convex direct sum of copies of $s \hat{\bigotimes}_{\varepsilon} E[1$, Theorem 4] (where $s$ denotes the space of all rapidly decreasing sequences). Hence $\mathcal{D}(e)$ has the approximation property since $s$ has it [5, (7), p. 284 and (2), p. 245]. 
2. The proof is similar to part 1 taking in account that $\mathcal{D}(e)$ has the strict approximation property since it is topologically isomorphic to $\mathcal{D}\left(\mathfrak{H}^{n}\right) \hat{\bigotimes}_{\varepsilon} E\left[9\right.$, prop. 9, p. 108] and $\mathcal{D}\left(\mathfrak{H}^{n}\right)$ has the strict approximation property [10, prop. 1, p. 6]. Applying [10, case 2, p. 48] we complete the proof.

Let $E$ be a strict $(L F)$-space, $p \in[1, \infty)$ and $\left(m_{i}\right)_{i \in \Perp}$ a strictly increasing sequence in $(0, \infty) \backslash \mathbf{N}$. We define $W^{\left(m_{i}\right) p}(E)=\bigcap_{i=1}^{\infty} W^{m_{i}, p}(E)$ and endowed it with the topology generated by $\left\{\|\|_{m_{i}, p}: i \in \mathbb{N}\right.$, $\|\cdot\| \in \operatorname{cs}(E)\}$.

COROLLARY 4.3 $W^{\left(m_{1}, \lambda\right)}(E)$ has the approximation property if $E$ has it.

PROOF. If $j \geq i$ denote by $I_{i j}: W^{m_{j}, p}(E) \rightarrow W^{m_{i}, p}(E)$ the canonical injection, here we use Lemma 3.1 , then $\left(W^{m_{i} P}(E), I_{i j}\right)$ is a projective sequence. Furthermore, $W^{m_{i, P}}(E)$ is topologically isomorphic to the projective limit of this sequence. Since $\mathcal{D}(E)$ is dense in each $W^{m_{i, P}}(E), \lim \left(W^{m_{i, P}}(E), I_{i j}\right)$ is a reduced projective limit and using Theorem 4.2 and $\left[5,(7)\right.$, p. 247] we have that $W^{\left(m_{i}\right) p}(E)$ has the approximation property.

\section{ACKNOWLEDGEMENT}

This work was made during the stay of J. Motos as a visiting professor at the Inst. Mat. U.N.A.M.

\section{REFERENCES}

[1] BONET, J. Representaciones de los espacios $\varepsilon^{k}(V, E) y \mathcal{D}^{k}(V, E)$, Rev. Real Acad. Ci. Madrid 76 (1982), 121-129.

[2] GARNIR, H. G., WILDE, M. de and SCHMETS, J. Analyse Fonctionnelle, Vol. 2, Birkhaüser, Basel-Stuttgart.

[3] GARNIR, H. G., WILDE, M. de and SCHMETS, J. Analyse Fonctionnelle, Vol. 3, Birkhaüser, Basel-Stuttgart.

[4] GROTHENDIECK, A. Sur certains espaces de fonctions holomorphes, Journal fïr die reine u. angew. Math. 192 (1953), 35-64 and 77-95.

[5] KÖTHE, G. Topological Vector Spaces II, Springer, Berlin, Heidelberg, New York, 1974.

[6] MOTOS, J. and PLANELLS, MA. J. Some locally convex properties of the vector valued Sobolev-Slobodeckij spaces $W^{m, p}(\Omega, E)$, Preprint 1988.

[7] MOTOS, J. and PLANELLS, MA. J. Una nota sobre los espacios de Sobolev anisotropos vectoriales $L_{p}^{p}$, Coll. Math, 38, 30 (1987), 249-256.

[8] PELCZYNSKI, A. and SENATOR, K. On isomorphisms of anisotropic Sobolev spaces with "classical Banach spaces" and a Sobolev type embedding theorem, Studia Math. 84 (1986), 169-215.

[9] SCHWARTZ, L. Espaces de fonctions differentiables à valeurs vectorielles, I. Analyse Math. 4 (1954-55), 88-148.

[10] SCHWARTZ, L. Théorie des distributions à valeurs vectorielles, Ch. I. Ann. Inst. Fourier Z (1957), 1-141.

[11] TRIEBEL, H. Interpolation Theory. Function Spaces. Differential Operators, North Holland, Amsterdam-New York, Oxford, 1978. 


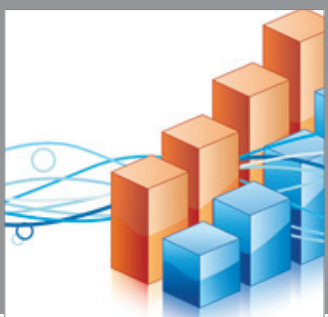

Advances in

Operations Research

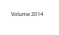

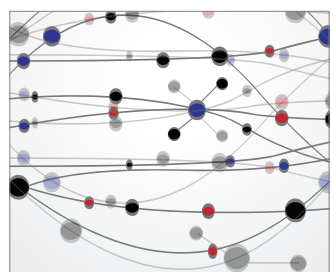

\section{The Scientific} World Journal
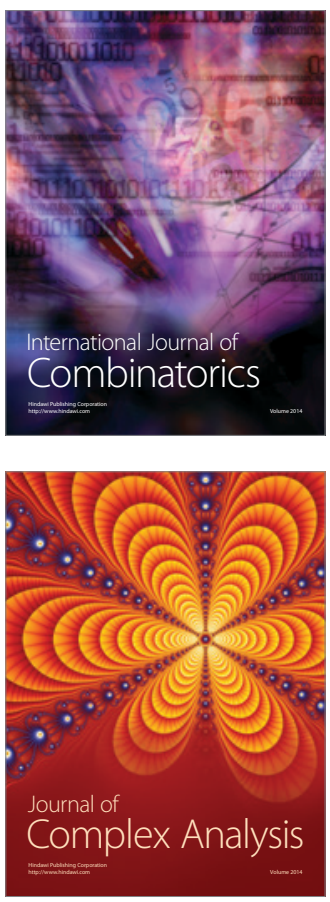

International Journal of

Mathematics and

Mathematical

Sciences
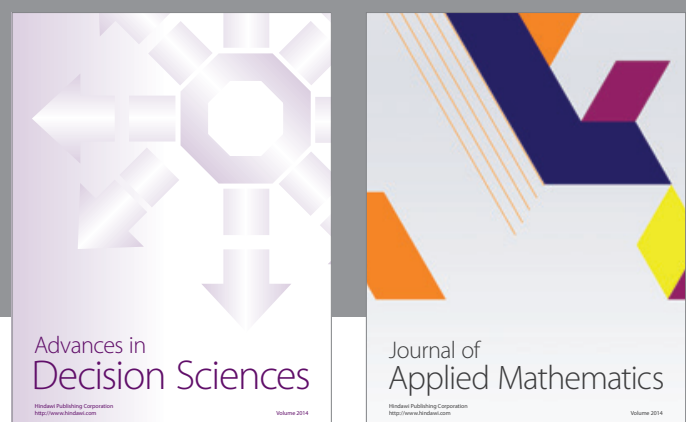

Journal of

Applied Mathematics
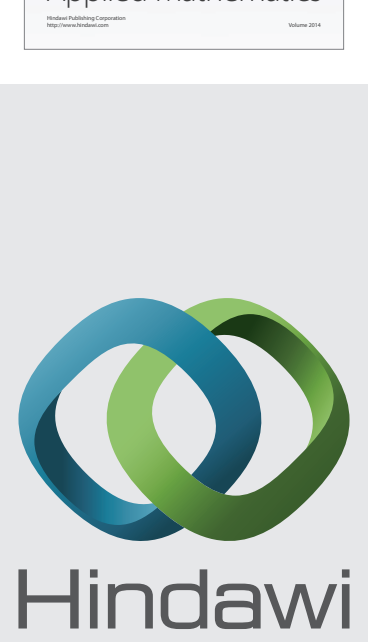

Submit your manuscripts at http://www.hindawi.com
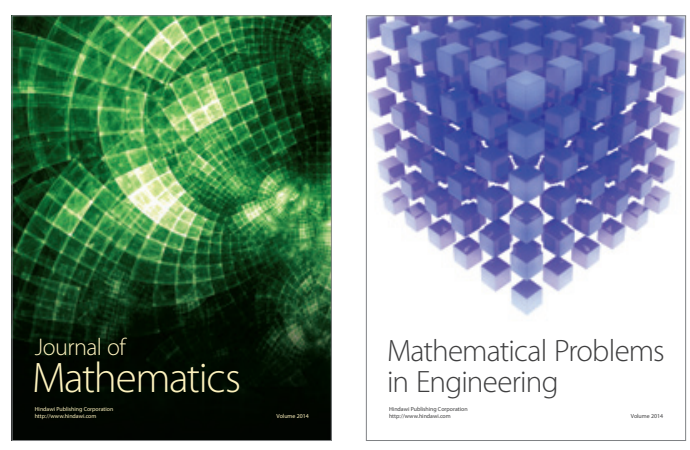

Mathematical Problems in Engineering
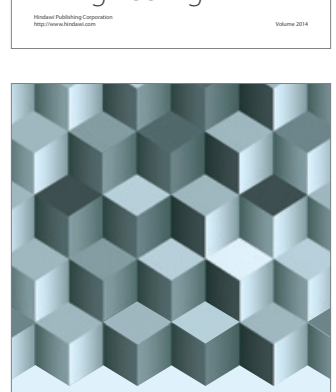

Journal of

Function Spaces
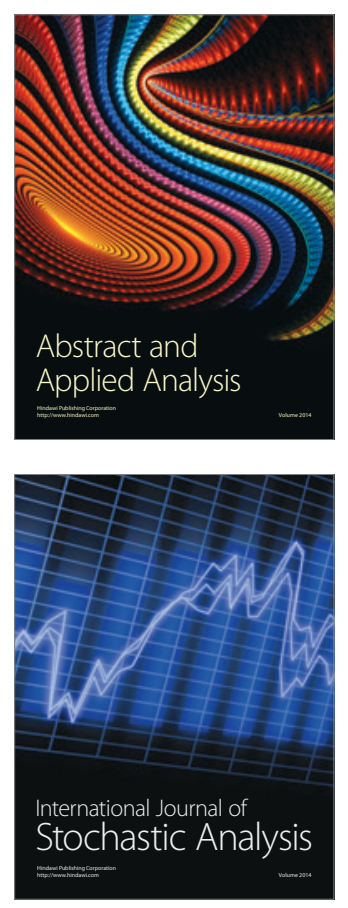

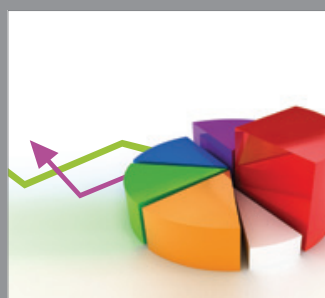

ournal of

Probability and Statistics

Promensencen
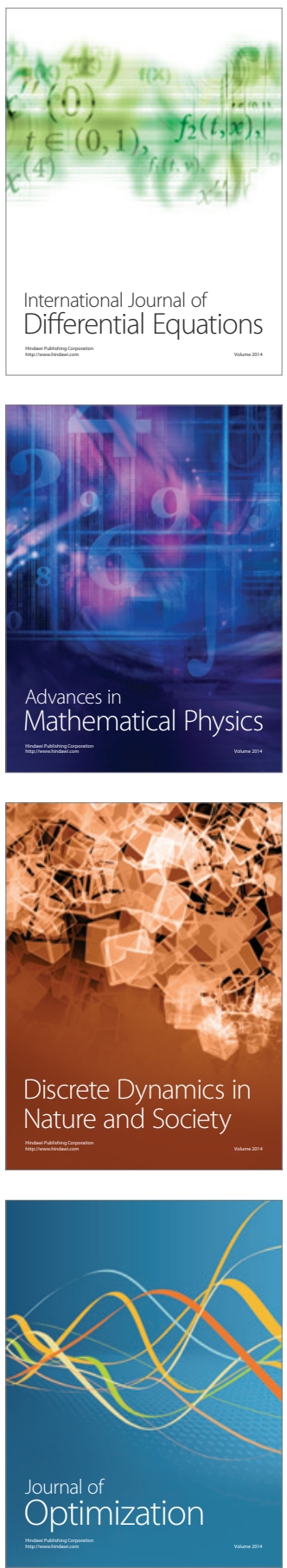